\title{
The role of acceleration and vorticity in relativistic hydro- dynamics
}

\author{
George Prokhorov ${ }^{1, *}$, Oleg Teryaev ${ }^{1,2,3, * *}$, and Valentin Zakharov ${ }^{2,4, * * *}$ \\ ${ }^{1}$ Joint Institute for Nuclear Research, 141980 Dubna, Russia \\ ${ }^{2}$ Institute of Theoretical and Experimental Physics, B. Cheremushkinskaya 25, 117218 Moscow, Russia \\ ${ }^{3}$ National Research Nuclear University MEPhI (Moscow Engineering Physics Institute), Kashirskoe \\ Shosse 31, 115409 Moscow, Russia \\ ${ }^{4}$ School of Biomedicine, Far Eastern Federal University, 690950 Vladivostok, Russia
}

\begin{abstract}
The role of vorticity and acceleration in relativistic hydrodynamics is investigated. Particular attention is paid to the effects of vorticity, as well as mixed effects, when the medium has both acceleration and vorticity. Quantumfield corrections to the energy density of free Dirac fields are calculated using the Zubarev density operator. The corresponding nonperturbative formulas are proposed and justified in the particular cases of parallel vorticity and acceleration and zero acceleration.
\end{abstract}

\section{Introduction}

Various quantum field effects associated with the rotation and acceleration of a medium are discovered. One of the most paradoxical quantum-field effect associated with acceleration is the Unruh effect [1], while the well-known quantum-field effect associated with vorticity is the chiral vortical effect [2].

These effects have so far been considered separately in different approaches. A remarkable observation of recent years is that they can be derived on the basis of a common fundamental theoretical approach based on the Zubarev density operator [3-5]. Moreover, this approach uses the standard perturbation theory in space with the Minkowski metric and there are no problems associated with the transition to a non-inertial frame. In particular, the calculation of corrections related to acceleration in the energy density made it possible to show the Unruh effect from the point of view of quantum statistical mechanics in an inertial frame [5].

In this paper, we continue to calculate corrections related to acceleration and vorticity. We calculate the corrections associated with the vorticity in the energy density of free Dirac field. First, the case of massless fields is considered, and then the results for massive fields are presented. Based on the obtained perturbative results, we substantiate the nonperturbative formula for a rotating fermion gas in general case of massive fermions. We also show that this nonperturbative formula can be strictly derived in the approach with the Wigner function [6].

\footnotetext{
*e-mail: prokhorov@theor.jinr.ru

**e-mail: teryaev@jinr.ru

***e-mail: vzakharov@itep.ru
} 
After that, we present the results of the calculation of corrections associated simultaneously with acceleration and vorticity. In this case we consider only massless fields. For the system with parallel acceleration and vorticity, we substantiate a nonperturbative formula, some of whose terms can be obtained from a less fundamental approach with the Wigner function.

An analysis of the obtained nonperturbative expressions allows one to obtain confirmation of the observation made in [7]. Namely, at least part of the effects of vorticity and acceleration is reduced to replacing the chemical potential $\mu \rightarrow \mu \pm|\omega| / 2 \pm i|a| / 2$, that is, vorticity contributes to the real part of the chemical potential, and acceleration to the imaginary part. Also polynomiality can be established as a consequence of nonperturbative formulas.

It is worth noting that in the particular case $a^{\mu}=0$, we obtain confirmation of the conclusion of [4] that all the effects of vorticity are reduced to replacing $\mu \rightarrow \mu \pm|\omega| / 2$. Moreover, this modification turns out to be valid in the general case of massive fermions. At the same time, only a part of the acceleration effects leads to the replacement of $\mu \rightarrow \mu \pm i|a| / 2$ and the generalization of this result to the case of particles with mass remains in question.

We discuss the general situation when acceleration is not parallel to vorticity. In this case, the polynomiality of the energy density is not obvious, and it is also not obvious what form the corresponding Sommerfeld integrals have. This situation is to be related to the fact that terms with boost and angular momentum operators in the density operator in this case does not commute with each other.

All results presented, except for section 2, are new and have not been previously published.

\section{Energy density of accelerated fermion gas}

The fundamental mathematical object describing a medium with acceleration and rotation, from the point of view of quantum statistical mechanics, is the Zubarev density operator [3]

$$
\hat{\rho}=\frac{1}{Z} \exp \left\{-\beta_{\mu}(x) \hat{P}^{\mu}+\frac{1}{2} \varpi_{\mu \nu} \hat{J}_{x}^{\mu v}+\zeta \hat{Q}\right\} .
$$

The effects associated with nonuniform motion - acceleration and rotation - are contained in a term with a thermal vorticity $\varpi$. In what follows, we will consider effects at zero chemical potential $\zeta=\mu / T=0$.

A perturbation theory based on (1) was developed and described in [3]. In [5], it was used to calculate corrections in the energy density of massless Dirac fields for an accelerated medium

$$
\rho=\frac{7 \pi^{2} T^{4}}{60}+\frac{T^{2}|a|^{2}}{24}-\frac{17|a|^{4}}{960 \pi^{2}}+O\left(|a|^{6}\right),
$$

where $|a|=\sqrt{-a_{\mu} a^{\mu}}$. One can also justify a more general non-perturbative formula $[5,8]$

$$
\rho=2 \int \frac{d^{3} p}{(2 \pi)^{3}}\left(\frac{|\mathbf{p}|+i|a|}{1+e^{\frac{|\mathbf{p}|}{T}+\frac{i|a|}{2 T}}}+\frac{|\mathbf{p}|-i|a|}{1+e^{\frac{|\mathbf{p}|}{T}-\frac{i|a|}{2 T}}}\right)+4 \int \frac{d^{3} p}{(2 \pi)^{3}} \frac{|\mathbf{p}|}{e^{\frac{2 \pi|\mathbf{p}|}{|a|}}-1},
$$

which exactly equal to (2) at $T>T_{U}$, and also receives additional motivation from the point of view of the Wigner function [6]. For $T<T_{U}$, according to (3) as it was shown in [5, 8], it turns out that the perturbation theory and the expression (2) are not applicable. This is due to the instability at $T=T_{U}$. A detailed discussion of this instability was given in [8].

It was shown that terms of higher orders $O\left(|a|^{6}\right)$ in (2) are equal to zero, as follows from a comparison with the method with a conical singularity and the properties of Sommerfeld integrals [8]. 
We also note that the generalization of the formula (3) to the case of massive particles is not obvious.

\section{Energy density of a rotating fermion gas}

\subsection{Massless Fields}

In the case of a rotating fermion gas, when vorticity $\omega^{\mu} \neq 0$ and $a^{\mu}=0$, the energy density in the fourth order of the perturbation theory has the following form

$$
\rho=A_{0} T^{4}+A_{1} T^{2}|\omega|^{2}+A_{2}|\omega|^{4}+O\left(|\omega|^{6}\right)
$$

where $|\omega|=\sqrt{-\omega_{\mu} \omega^{\mu}}$. The coefficients $A_{1}$ and $A_{2}$ can be calculated using (1). The procedure of calculation of similar coefficients is described in [3]. In particular, the coefficient $A_{0}$ corresponds to the standard expression for the fermion gas energy in statistical physics, and $A_{1}$ was calculated in [3]. The fourth-order coefficient $A_{2}$ is expressed in terms of a quantity of the form [5]

$$
\begin{aligned}
& C^{\alpha_{1} \alpha_{2}\left|\alpha_{3} \alpha_{4}\right| \alpha_{5} \alpha_{6}\left|\alpha_{7} \alpha_{8}\right| \alpha_{9} \alpha_{10} \mid i j k l}=\int_{0}^{\beta \mid} d \tau_{x} d \tau_{y} d \tau_{z} d \tau_{f} d^{3} x d^{3} y d^{3} z d^{3} f \\
& \times x^{i} y^{j} z^{k} f^{l}\left\langle T_{\tau} \hat{T}^{\alpha_{1} \alpha_{2}}\left(\tau_{x}, \mathbf{x}\right) \hat{T}^{\alpha_{3} \alpha_{4}}\left(\tau_{y}, \mathbf{y}\right) \hat{T}^{\alpha_{5} \alpha_{6}}\left(\tau_{z}, \mathbf{z}\right) \hat{T}^{\alpha_{7} \alpha_{8}}\left(\tau_{f}, \mathbf{f}\right) \hat{T}^{\alpha_{9} \alpha_{10}}(0)\right\rangle_{\beta(x), c} .
\end{aligned}
$$

By choosing an appropriate reference frame and vorticity components, we can show that

$$
\begin{aligned}
& A_{2}=\frac{1}{4 !}\left(C^{02|02| 02|02| 00 \mid 1111}-C^{02|02| 02|01| 00 \mid 1112}-C^{02|02| 01|02| 00 \mid 1121}+C^{02|02| 01|01| 00 \mid 1122}\right. \\
& -C^{02|01| 02|02| 00 \mid 1211}+C^{02|01| 02|01| 00 \mid 1212}+C^{02|01| 01|02| 00 \mid 1221}-C^{02|01| 01|01| 00 \mid 1222} \\
& -C^{01|02| 02|02| 00 \mid 2111}+C^{01|02| 02|01| 00 \mid 2112}+C^{01|02| 01|02| 00 \mid 2121}-C^{01|02| 01|01| 00 \mid 2122} \\
& \left.+C^{01|01| 02|02| 00 \mid 2211}-C^{01|01| 02|01| 00 \mid 2212}-C^{01|01| 01|02| 00 \mid 2221}+C^{01|01| 01|01| 00 \mid 2222}\right)
\end{aligned}
$$

Calculation of quantum correlators at finite temperature in (6) in the case of massless fields $m=0$ gives the following answer

$$
A_{2}=\frac{1}{64 \pi^{2}}
$$

Thus, the energy density of the accelerated fermion gas has the form

$$
\rho=\frac{7 \pi^{2} T^{4}}{60}+\frac{T^{2}|\omega|^{2}}{8}+\frac{|\omega|^{4}}{64 \pi^{2}}+O\left(|\omega|^{6}\right) .
$$

\subsection{Massive Fields}

In the general case of massive fermions, the following expression was obtained for the coefficient $A_{2}$ from (6)

$$
A_{2}=\frac{1}{192 \pi^{2}} \int_{0}^{\infty} d p p^{2} E_{p} n_{F}^{\prime \prime \prime \prime}\left(E_{p}\right),
$$

where $n_{F}\left(E_{p}\right)=1 /\left(e^{E_{p} / T}+1\right)$ is the Fermi distribution, $n_{F}^{\prime \prime \prime \prime}\left(E_{p}\right)=\frac{d^{4}}{d E_{p}^{4}} 1 /\left(e^{E_{p} / T}+1\right)$ is a fourth order derivative, and $E_{p}=\sqrt{m^{2}+|\mathbf{p}|^{2}}$. The coefficients $A_{0}$ and $A_{1}$ were previously calculated in [3] in general case at $m \neq 0$. 
The energy density of the accelerated fermion gas can also be obtained using the Wigner function [6]. The procedure for calculating nonperturbative mean values using the Wigner function is described in [9]. For the energy density, the following expression is obtained

$$
\rho=2 \int \frac{d^{3} p}{(2 \pi)^{3}} E_{p}\left(\frac{1}{1+e^{\frac{E_{p}}{T}+\frac{|\omega|}{2 T}}}+\frac{1}{1+e^{\frac{E_{p}}{T}-\frac{|\omega|}{2 T}}}\right) .
$$

All mass effects in (10) are contained in terms with $E_{p}$. If we now consider (10) perturbatively, we can obtain the expansion in $|\omega|$ and calculate the terms $|\omega|^{2},|\omega|^{4}$, which then can be compared with the coefficients $A_{0}, A_{1}, A_{2}$, calculated using the density operator (1). It is amusing, that the coefficients obtained in such a way exactly correspond to the fundamental calculation based on the density operator. This situation is completely analogous to the case with axial current [4].

Thus, the formula (10) is a very good candidate for the role of a nonperturbative expression for the energy density in a system with rotation in the general case of massive fermions. The analysis (10) confirms the conclusion of [7] that the vorticity plays the role of the real chemical potential $\mu \rightarrow \mu \pm|\omega| / 2$.

It should be noted that in the chiral limit the integrals in (10) are the polynomial Sommerfeld integrals, which can be shown by integration in the complex plane [8] and therefore the corrections $O\left(|\omega|^{6}\right)$ in (4) are equal to 0 and the formula (4) is exact.

\section{Energy density of a fermion gas with acceleration and rotation}

\subsection{Perturbative corrections in the general case}

In the case when there is simultaneously vorticity and acceleration, the energy density takes the form

$$
\rho=A_{0} T^{4}+A_{1} T^{2}|\omega|^{2}+A_{2}|\omega|^{4}+A_{3} T^{2}|a|^{2}+A_{4}|a|^{4}+A_{5}|a|^{2}|\omega|^{2}+A_{6}(\omega a)^{2}+O\left(\varpi^{6}\right),
$$

where $(\omega a)=\omega_{\mu} a^{\mu}$ is the scalar product. The terms $A_{0}, A_{1}, A_{3}$ were calculated in [3], the terms $A_{2}, A_{4}$ were presented in the previous sections, and the terms $A_{5}, A_{6}$ should be calculated. They can be calculated using formulas similar to the formulas from the previous section. As a result, we get

$$
\rho=\frac{7 \pi^{2} T^{4}}{60}+\frac{T^{2}|\omega|^{2}}{8}+\frac{|\omega|^{4}}{64 \pi^{2}}+\frac{T^{2}|a|^{2}}{24}-\frac{17|a|^{4}}{960 \pi^{2}}+\frac{23|a|^{2}|\omega|^{2}}{1440 \pi^{2}}+\frac{11(\omega a)^{2}}{720 \pi^{2}}+O\left(\varpi^{6}\right) .
$$

In the particular case of parallel acceleration and vorticity, when $(\omega a)^{2}=|a|^{2}|\omega|^{2}$, we have

$$
\rho=\frac{7 \pi^{2} T^{4}}{60}+\frac{T^{2}|\omega|^{2}}{8}+\frac{|\omega|^{4}}{64 \pi^{2}}+\frac{T^{2}|a|^{2}}{24}-\frac{17|a|^{4}}{960 \pi^{2}}+\frac{|a|^{2}|\omega|^{2}}{32 \pi^{2}}+O\left(\varpi^{6}\right) .
$$

\subsection{Nonperturbative formula in the case of parallel $a$ and $\omega$}

In the case of parallel acceleration and vorticity, $(\omega a)^{2}=|a|^{2}|\omega|^{2}$, by analogy with (3) and (10), we can write the integral representation

$$
\begin{aligned}
\rho= & \int \frac{d^{3} p}{(2 \pi)^{3}}\left(\frac{|\mathbf{p}|+i|a|}{1+e^{\frac{|\mathbf{p}|}{T}+\frac{|\omega|}{2 T}+\frac{i|a|}{2 T}}}+\frac{|\mathbf{p}|-i|a|}{1+e^{\frac{|\mathbf{p}|}{T}+\frac{|\omega|}{2 T}-\frac{i|a|}{2 T}}}+\frac{|\mathbf{p}|+i|a|}{1+e^{\frac{|\mathbf{p}|}{T}-\frac{\omega \omega \mid}{2 T}+\frac{|a|}{2 T}}}+\frac{|\mathbf{p}|-i|a|}{1+e^{\frac{\mid \mathbf{p}}{T}-\frac{|\omega|}{2 T}-\frac{i|a|}{2 T}}}\right) \\
& +4 \int \frac{d^{3} p}{(2 \pi)^{3}} \frac{|\mathbf{p}|}{e^{\frac{2 \pi|\mathbf{p}|}{|a|}}-1} .
\end{aligned}
$$


Eq. (14) exactly coincides with (13) for $T>T_{U}$, where $T_{U}=|a| /(2 \pi)$ is the Unruh temperature. Moreover, some of the terms is reproduced using the Wigner function, where the following expression is obtained

$$
\rho=\int \frac{d^{3} p|\mathbf{p}|}{(2 \pi)^{3}}\left(\frac{1}{1+e^{\frac{|p|}{T}+\frac{g_{\omega}}{2 T}+\frac{i g_{a}}{2 T}}}+\frac{1}{1+e^{\frac{|\mathbf{p}|}{T}+\frac{q_{\omega}}{2 T}-\frac{i g_{a}}{2 T}}}+\frac{1}{1+e^{\frac{|\mathbf{p}|}{T}-\frac{g_{\omega}}{2 T}+\frac{i g_{a}}{2 T}}}+\frac{1}{1+e^{\frac{|\mathbf{p}|}{T}-\frac{g_{\omega}}{2 T}-\frac{i g_{a}}{2 T}}}\right)
$$

where the specific form of $g_{a}$ and $g_{\omega}$ is given in [7]. In particular, $g_{a}$ and $g_{\omega}$ equal to $|a|$ and $|\omega|$ in the particular case of parallel $a$ and $\omega$.

Let us analyze the consequences of (14). According to (14) the vorticity contributes to the real part of the chemical potential, and the acceleration contributes to the imaginary part, and thus there is a replacement $\mu \rightarrow \mu \pm|\omega| / 2 \pm i|a| / 2$. But the effects of acceleration are not limited to substitutions in the chemical potential. Also, apparently, the replacement $\mu \rightarrow \mu \pm|\omega| / 2 \pm i|a| / 2$ is valid only in the case of parallel $a$ and $\omega$, as follows from a comparison with (15), where more general combinations $g_{a}$ and $g_{\omega}$ appear, which reduce to $|a|$ and $|\omega|$ only in the particular case of parallel $a$ and $\omega$.

It also follows from (14) that the expression (13) is exact, which can be shown by analogy with how it was done in [8] by integration in the complex plane.

\section{Conclusions}

Corrections related to the vorticity in the energy density of Dirac free fields are calculated. The case of massless fields and the general case of massive fields are considered. The nonperturbative formula for energy with vorticity is substantiated. This formula is exactly confirmed by the calculation based on the Wigner function, and also in the first four orders exactly coincides with the prediction of perturbative fundamental calculation.

Based on the obtained formula, one can substantiate the concept of vorticity as a real chemical potential, which was previously observed for axial current [7]. This nonperturbative formula allows one to substantiate a polynomial form of energy density with vorticity in the chiral limit, where the maximum order is four.

In the case of massless fields, mixed effects are investigated, when there is both acceleration and vorticity. In case of parallel acceleration and vorticity, a nonperturbative formula for the energy density in the form of Sommerfeld integrals is substantiated.

It follows from this formula that in case of parallel acceleration and vorticity, they contribute to the chemical potential according to the relation $\mu \rightarrow \mu \pm|\omega| / 2 \pm i|a| / 2$, although, unlike vorticity, the effects of acceleration are not reduced only to such a replacement of chemical potential. In the more general case of non-parallel acceleration and vorticity, a more complicated situation takes place.

\section{Acknowledgements}

The reported study was funded by RFBR according to the research projects 18-02-40056 and 17-02-01108.

\section{References}

[1] W. G. Unruh, Phys. Rev. D 14, 870 (1976). doi:10.1103/PhysRevD.14.870

[2] D. E. Kharzeev, K. Landsteiner, A. Schmitt and H. U. Yee, Lect. Notes Phys. 871 (2013).

[3] M. Buzzegoli, E. Grossi and F. Becattini, JHEP 1710 (2017) 091.

[4] G. Y. Prokhorov, O. V. Teryaev and V. I. Zakharov, JHEP 1902, 146 (2019).

[5] G. Y. Prokhorov, O. V. Teryaev and V. I. Zakharov, Phys. Rev. D 99, no. 7, 071901 (2019). 
[6] F. Becattini, V. Chandra, L. Del Zanna and E. Grossi, Annals Phys. 338 (2013) 32.

[7] G. Prokhorov, O. Teryaev and V. Zakharov, Phys. Rev. D 98, no. 7, 071901 (2018).

[8] G. Y. Prokhorov, O. V. Teryaev and V. I. Zakharov, arXiv:1906.03529 [hep-th].

[9] G. Prokhorov and O. Teryaev, Phys. Rev. D 97, no. 7, 076013 (2018) doi:10.1103/PhysRevD.97.076013 [arXiv:1707.02491 [hep-th]]. 